\title{
Nano rough micron patterned titanium for directing osteoblast morphology and adhesion
}

\author{
Sabrina Puckett \\ Rajesh Pareta \\ Thomas J Webster \\ Division of Engineering, Brown \\ University, Providence, RI, USA
}

Correspondence:Thomas J Webster Divisions of Engineering and Orthopedics, Brown University, Providence, RI 02917, USA

Tel +l 40I 5233802

Fax + I 40I 5239107

Email thomas_webster@brown.edu

\begin{abstract}
Previous studies have demonstrated greater functions of osteoblasts (bone-forming cells) on nanophase compared with conventional metals. Nanophase metals possess a biologically inspired nanostructured surface that mimics the dimensions of constituent components in bone, including collagen and hydroxyapatite. Not only do these components possess dimensions on the nanoscale, they are aligned in a parallel manner creating a defined orientation in bone. To date, research has yet to evaluate the effect that organized nanosurface features can have on the interaction of osteoblasts with material surfaces. Therefore, to determine if surface orientation of features can mediate osteoblast adhesion and morphology, this study investigated osteoblast function on patterned titanium substrates containing alternating regions of micron rough and nano rough surfaces prepared by novel electron beam evaporation techniques. This study was also interested in determining whether or not the size of the patterned regions had an effect on osteoblast behavior and alignment. Results indicated early controlled osteoblast alignment on these patterned materials as well as greater osteoblast adhesion on the nano rough regions of these patterned substrates. Interestingly, decreasing the width of the nano rough regions (from $80 \mu \mathrm{m}$ to $22 \mu \mathrm{m}$ ) on these patterned substrates resulted in a decreased number of osteoblasts adhering to these areas. Changes in the width of the nano rough regions also resulted in changes in osteoblast morphology, thus, suggesting there is an optimal pattern dimension that osteoblasts prefer. In summary, results of this study provided evidence that aligned nanophase metal features on the surface of titanium improved early osteoblast functions (morphology and adhesion) promising for their long term functions, criteria necessary to improve orthopedic implant efficacy.
\end{abstract}

Keywords: osteoblasts, titanium, nanophase, orthopedic, alignment, surface topography

\section{Introduction}

Orthopedic implant devices are becoming a widespread resource for improving the quality of life for all individuals. In 2004, the number of patients receiving a total hip replacement for the first time was 234,000 (AAOS 2006a) and the number of patients receiving a total knee replacement for the first time was 455,000 (AAOS 2006b). The demand for such orthopedic implant devices is continuing to rise drastically. In fact, there will be a $174 \%$ increase for first time total hip replacements and a $673 \%$ increase for first time total knee replacements by the year 2030 (AAOS 2006c). Such statistics do not include the steadily growing number of revision surgeries which is due to the fact that the average lifespan of an orthopedic implant is only 10 to 15 years. The number of revision surgeries for total hip replacements by 2026 and total knee replacements is expected to double by 2015 (AAOS 2006c). Clearly, more emphasis needs to be placed on finding ways to expand the longevity of orthopedic implants since current methods are neither reliable nor sufficient.

Because the implant surface is in direct contact with living tissue, interactions between the device and its surroundings must be favorable. Unfortunately, current orthopedic implant surfaces are not reacting successfully to living tissues, such as 
bone. More specifically, the lack of appropriate cell adhesion (the physiochemical linkage and protein interaction between cells and the implant surface; Anselme 2000; Jayaraman et al 2004) and the lack of osseointegration (the bonding between the implant and surrounding bone; Brunski 1991; Webster 2001) are clinical complications that lead to short lifespans of current orthopedic implants. Osseointegration is specifically needed to ensure successful implantation because it involves the growth of bone tissue to the surgically implanted device providing a secure and strong attachment. By increasing implant stability and minimizing damage caused by the movement of the implant, the overall success rate and effectiveness of the implant is improved.

Due to the growing need for improvements in orthopedic implant devices, modifications of the implant surface is one way to maximize bonding to juxtaposed bone. In fact, the surface properties of current bone implants have already been modified in a way to better mimic the surface roughness of natural bone. Bone consists mainly of a fibrous, organic matrix composed of $90 \%$ type 1 collagen and an inorganic matrix composed mainly of hydroxyapatite. Type I collagen is synthesized by osteoblasts and contains linear fibrils $300 \mathrm{~nm}$ in length and $0.5 \mathrm{~nm}$ in diameter (Webster 2001). Hydroxyapatite crystals have a hexagonal unit cell structure and can range from 2-5 nm thick and 20-80 nm long (Rho et al 1998). Together, these chemical components in the bone matrix form a nanotextured surface that is responsible for further promoting osteoblast functions and for the unique mechanical properties (such as toughness) of bone. Recent studies have demonstrated that the use of nanophase metals, especially titanium and titanium alloys, enhance bone formation and growth when compared with conventional metals because the nanophase metals better mimic these characteristic dimensions of bone constituents (Webster and Ejiofor 2004; Yao et al 2005; Ward and Webster 2007). As a result of recreating the natural nanostructure of bone, nanophase materials (including nanophase ceramics, metals, polymers, and composites) decrease infection, decrease inflammation, and promote osseointegration (Liu and Webster 2007), all of which improve the success and longevity of orthopedic implants.

Further examining the hierarchical structure of bone reveals that collagen spontaneously forms fibrils of aligned nanoscale protein helices (Holmgren et al 1998; Lodish et al 2000; Taton 2001). These fibers serve as a template for the nucleation and growth of hydroxyapatite crystals, thereby creating a highly organized nanostructured surface that exists in a parallel orientation to the physiological loading of bone.
Therefore, the size and orientation of the hydroxyapatite crystals depend directly on the organization of the collagen fibril helices. The collagen fibrils are also responsible for providing binding sites for undifferentiated bone-marrow stem cells that develop into osteoblasts and promote bone growth. Clearly, creating surfaces that incorporate these aligned nano structural features similar to natural bone could be further design criteria used to extend the lifetime of orthopedic implants.

For this reason, the objectives of the present in vitro study were to fabricate titanium with biologically inspired linear nano features similar to both the size and orientation of collagen fibrils and hydroxyapatite in bone and explore early osteoblast functions on these surfaces. This study further examined whether or not osteoblast function was affected by the size of the linear nanophase features in order to determine the optimal dimensions that support early osteoblast functions.

\section{Materials and methods Substrate preparation}

Cell adhesion studies were performed on commercially pure titanium (Ti) substrates obtained from Alfa Aesar (Ward Hill, MA, USA) and borosilicate glass obtained from Fisher Scientific (Agawam, MA, USA). The borosilicate glass substrates served as the control in the cell experiments. The glass substrates were rinsed ultrasonically in acetone and ethanol successively for 10 minutes each. After rinsing, the glass substrates were etched in $1 \mathrm{~N} \mathrm{NaOH}$ for 1 hour and rinsed thoroughly in distilled water several times. The $\mathrm{Ti}$ substrates, with dimensions of $10 \mathrm{~mm} \times 10 \mathrm{~mm} \times 2 \mathrm{~mm}$, were rinsed ultrasonically in acetone, ethanol, and distilled water successively for 10 minutes each and dried in an oven at $40{ }^{\circ} \mathrm{C}$ for 15 minutes. These Ti substrates served as the template for the entire micron rough surface, entire nano rough surface, and patterned substrates (alternating regions of micron and nano roughness). All substrates were sterilized at $48.9^{\circ} \mathrm{C}$ and $138 \mathrm{kPa}$ in a steam autoclave for 30 minutes before being used in cell experiments.

\section{Entire micron rough substrates}

The substrates received from the factory contained micron rough features. Therefore, no preparation, other than sterilization, was required to create a substrate containing an entire micron rough nsurface.

\section{Patterned substrates}

The patterned substrates were created using a Temescal Electron Beam Evaporator and copper grids (Figure 1) 


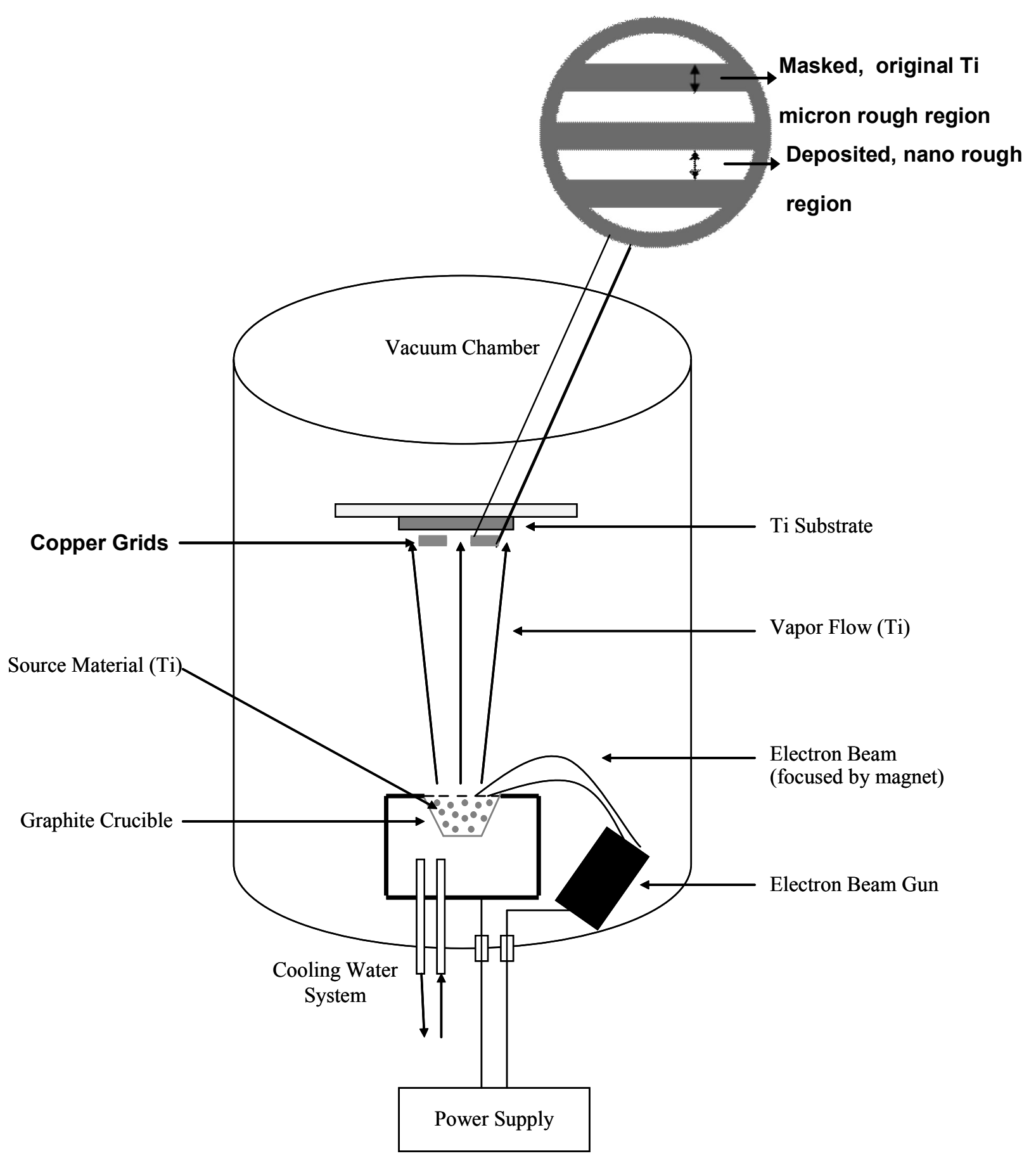

Figure I Schematic diagram of the electron beam evaporation technique used to create novel nanopatterned micron regions on Ti. Copper grids are magnified to indicate how the linear micron rough and nano rough regions are created.

obtained from Electron Microscopy Sciences (Hatfield, PA, USA). Electron beam evaporation involves concentrating large amounts of heat produced by high energy electron beam bombardment on the source material to be deposited, in this case $99.995 \%$ pure Ti (Kamis; Mahopac Falls, NY, USA). The electron beam is generated by an electron gun that uses the thermoionic emission of electrons produced by an incandescent filament. A magnet focuses and bends the electron trajectory so that the beam is accelerated towards a graphite crucible (Lesker Co; Clairton, PA, USA) containing the source material. As the beam rotates and hits the surface of the source material, heating and vaporization occur enabling the atoms and molecules to evaporate freely throughout the vacuum chamber. The atoms and molecules 
in the vapor flow then condense onto the substrate surface located at the top of the chamber creating a coating that masks the original surface. In this study, Ti was deposited onto the Ti substrates at a rate of $3.0 \AA /$ s and at a thickness of $500 \mathrm{~nm}$. The deposition rate is a parameter that controls the rate of grain growth, and $3.0 \AA / \mathrm{s}$ creates a coating containing nano size grains.

The patterned substrates possessed a linear configuration of alternating micron rough and nano rough features by attaching copper grids onto the Ti substrate before being placed into the electron beam evaporator. The opening (hole) in the grids was the area in which the Ti was deposited to create a coating containing nano rough features while the portions containing the copper (bar) camouflaged the original $\mathrm{Ti}$ surface during this deposition to maintain the original micron rough Ti topography. The grids varied in their dimensions (both the size of the opening where the original surface was exposed and the area where the original surface was camouflaged) allowing for the control of nano and micron rough regions. In this study, three different types of copper grids were used: 1) the nano deposited width (opening) was $80 \mu \mathrm{m}$ and the micron width (bar) was $45 \mu \mathrm{m}, 2$ ) the nano deposited width was $48 \mu \mathrm{m}$ and the micron width was $35 \mu \mathrm{m}$, and 3) the nano deposited width was $22 \mu \mathrm{m}$ and the micron width was $30 \mu \mathrm{m}$. After deposition, the grids were removed, and the substrates were rinsed in acetone, ethanol, and distilled water successively for 10 minutes each and dried in an oven for 15 minutes at $40{ }^{\circ} \mathrm{C}$.

\section{Entire nano rough substrates}

The entire nano rough surfaces were also created using the electron beam evaporator. However, no grids were placed on the substrate, thus, exposing the whole surface to the vapor flow that condensed on the surface. As a result, a deposited layer of nano grains formed over the entire original Ti surface changing the overall roughness of the substrate. After deposition, the substrates were rinsed in acetone, ethanol, and distilled water successively for 10 minutes each and dried in an oven for 15 minutes at $40{ }^{\circ} \mathrm{C}$.

\section{Material characterization}

A LEO 1530VP scanning electron microscope (SEM) was used to qualitatively characterize the surfaces of the patterned Ti substrates. Specifically, SEM was used to indicate the distinction in roughness of the two regions and that these varying surface topographies were maintained upon removal of the copper grids as well as after cleaning and autoclaving the samples. Images were created using secondary electrons collected with the in-lens detector at a $3 \mathrm{kV}$ acceleration voltage.

A Park Scientific Autoprobe CP Atomic Force Microscope was used to quantitatively evaluate the surface topography of the patterned Ti substrates. The AFM measured changes in surface area and root mean square (rms) roughness. Contact mode was used to capture the height images of each sample. The analyzed field was $2 \mu \mathrm{m} \times 2 \mu \mathrm{m}$ at a scan rate of $1 \mathrm{~Hz}$. PSI ProScan Image Processing software was used to analyze the resulting images. The surface area and root mean squared (rms) surface roughness (nm) in four random, arbitrary fields per substrate were obtained, averaged, and recorded.

A diffraktometer D500 Siemens (XRD) was used to characterize the crystalline phase and chemistry of the patterned Ti substrates. XRD was used in order to test another parameter that could affect cell behavior on these surfaces (crystalline phase). Spectra were taken using a power supply of $30.0 \mathrm{~mA}$ and $40.0 \mathrm{kV}$.

\section{Cell culture}

Commercially available human osteoblasts (bone-forming cells, CRL-11372, American Type Culture Collection, population numbers 6-9) were cultured in Dulbecco's Modified Eagles Medium (DMEM, Hyclone; Logan, UT, USA) supplemented with $10 \%$ fetal bovine serum (FBS, Hyclone) and $1 \%$ penicillin-streptomycin $(\mathrm{P} / \mathrm{S}$, Hyclone) under standard cell culture conditions $\left(5 \% \mathrm{CO}_{2} / 95 \%\right.$ humidified air environment at $37^{\circ} \mathrm{C}$ ).

\section{Cell adhesion}

Osteoblasts were enzymatically lifted from the tissue culture polystyrene culture dish using $1 \mathrm{~mL}$ of TrypsinEDTA 1x solution (Sigma Aldrich; Milwaukee, WI, USA). Osteoblasts were then suspended in DMEM supplemented with $10 \% \mathrm{FBS}$ and $1 \% \mathrm{P} / \mathrm{S}$ and were separately seeded at a density of 3500 cells $/ \mathrm{cm}^{2}$ onto the substrates of interest in a standard 24-well culture plate under standard cell conditions $\left(5 \% \mathrm{CO}_{2} / 95 \%\right.$ humidified air environment at $37^{\circ} \mathrm{C}$ ) for 4 hours. After this time period, substrates were rinsed in phosphate buffered saline (PBS) to remove any non-adherent cells. Adherent cells on the substrates were fixed with formalin based acetate buffer (Fisher Scientific), stained with DAPI dilactate (Sigma Aldrich), and counted under a Zeiss Axiovert $200 \mathrm{M}$ fluorescent microscope. Images were captured using an Orca ER camera. Rhodamine phalloidin (Invitrogen; Carlsbad, CA, USA) was used to examine the f-actin filaments in the cytoskeleton of the cells 
under fluorescence microscopy. The DAPI dilactate and rhodamine phalloidin experiments were completed simultaneously. All experiments were run in duplicate and repeated at least three times.

Osteoblast morphology and adhesion location on the substrates of interest to this study were examined using a LEO 1530VP SEM. At the end of 4 hours, the cells were fixed with formalin based acetate buffer (Fisher Scientific) and dehydrated through sequential washings in $10 \%, 30 \%$, $50 \%, 70 \%, 90 \%$, and 100\% ethanol (remainder deionized water) solutions. Samples were then critically point dried using a Ladd Research Critical Point Dryer under standard conditions. Finally, samples were sputter-coated with a thin layer of gold-palladium using an ISI PS-2 Coating Unit in a $100 \mathrm{mT}$ Torr vacuum argon environment for 3 minutes and $10 \mathrm{~mA}$ of current. Images were created using secondary electrons collected with the in-lens detector at a $3 \mathrm{kV}$ acceleration voltage.

\section{Statistical analysis}

Data was analyzed using standard analysis of variance (ANOVA) techniques. Statistical significance was considered at $\mathrm{p}<0.05$.

\section{Results}

\section{Surface characterization}

Results from SEM confirmed the alternating regions of nanometer surface roughness and micron surface roughness on Ti substrates produced by electron beam evaporation (Figure 2). The original Ti surfaces displayed micron rough characteristics as they appeared more smooth and flat when compared to the modified regions at SEM $200 \mathrm{~nm}$ scale bar magnifications. On the other hand, these modified, deposited areas at the same magnification exhibited more nanometer features as they contained a large number of nanoscale grains homogenously distributed over the region. As a result, the overall surface roughness and surface area increased on the regions patterned by electron beam evaporation in relation to the original $\mathrm{Ti}$ surface that was masked during the procedure.

Results from AFM confirmed the varying surface topography on the patterned Ti substrates as indicated by SEM (Figure 3). The deposited regions on the patterned substrates clearly displayed nano rough characteristics due to the presence of more nanoscale bumps. The unmodified regions lack the rough terrain and contain more conventional characteristics due to the larger, micron size dips and ridges. The measured rms value for the nano rough regions was
$20 \mathrm{~nm}$ while the rms value for the micron rough region was $56.75 \mathrm{~nm}$. Moreover, the average surface area increased due to $\mathrm{z}$-direction roughness $91.70 \%$ for the nano rough regions and $80.20 \%$ for the micron rough regions.

XRD images confirmed there was a slight difference in the crystalline phase of the two regions of the patterned substrates (Figure 4). More specifically, the results indicated that the unmodified, conventional region contained rutile $\mathrm{TiO}_{2}$ and no anatase $\mathrm{TiO}_{2}$. On the other hand, the results for the modified, nano region showed anatase $\mathrm{TiO}_{2}$ and no rutile $\mathrm{TiO}_{2}$. Clearly, the Ti material deposited onto these surfaces had a different crystallinity than the original Ti surface.

\section{Osteoblast adhesion}

Results of the present study revealed that after 4 hours, osteoblast adhesion increased on the entire nano rough $\mathrm{Ti}$ surface that was modified through electron beam evaporation compared to the entire micron rough Ti surface that was not altered (Figure 5). In fact, osteoblast adhesion was significantly greater $(\mathrm{p}<0.01)$ on the substrates that were completely modified to contain nano rough features compared to the unmodified micron rough substrates and the patterned Ti substrate containing both micron and nano rough regions. Results of increased osteoblast adhesion on nanophase compared with conventional Ti confirmed those of previous studies where the nano features were created using different techniques, such as anodization and particle compaction (Webster and Ejiofor 2004; Yao et al 2005). Furthermore, when examining the three grid types, results indicated that the total number of osteoblasts adhered over the entire patterned substrates (including both micron and nano regions) were not affected by varying the width of the modified nano rough region (Figure 5). That is, total osteoblast adhesion over the entire surface of the patterned substrates showed no significant difference among the varying dimensions (or the entire unmodified micron rough Ti surface). As a result, total osteoblast density on these surfaces was not a direct result of size of the deposited nano rough region. Of course, even though the total numbers were similar, the distribution of these cells between nano and micron rough regions may be different.

Interestingly, when analyzing the specific location of cells (the region of attachment) on each of the three patterned $\mathrm{Ti}$ substrates, greater osteoblast adhesion was detected on the deposited nano rough Ti region compared to the original micron rough region regardless of width size (Figure 6). This suggests that surface roughness is being identified by osteoblasts who are favoring the surfaces exhibiting more 
nano characteristic dimensions. Upon further examination of the osteoblasts adhered to the nano rough portion of these patterned substrates, data indicated that decreasing the width of the nano rough region resulted in decreased osteoblast adhesion in the nano rough region (Figures 6 and 7). In other words, osteoblast adhesion was significantly greater ( $p<0.05$ and 0.01 ) on the Ti substrate containing the $80 \mu \mathrm{m}$ nano rough width spacings compared to those substrates containing the 48 and $22 \mu \mathrm{m}$ nano rough width spacings. This could be due in part to cell size. More specifically, the average size of an osteoblast is $20-30 \mu \mathrm{m}$, and therefore, the cell may be unable to fit and attach properly within the small nano rough regions on the patterned Ti substrates, especially those containing the $22 \mu \mathrm{m}$ width spacings.

\section{Osteoblast morphology}

The present study not only provided evidence of the differences in overall osteoblast adhesion but early signs of osteoblast alignment on the patterned Ti substrates was also detected after just 4 hours of cell culture. In fact, osteoblast morphology on the patterned $\mathrm{Ti}$ substrates, regardless of width size, demonstrated an elongated orientation when

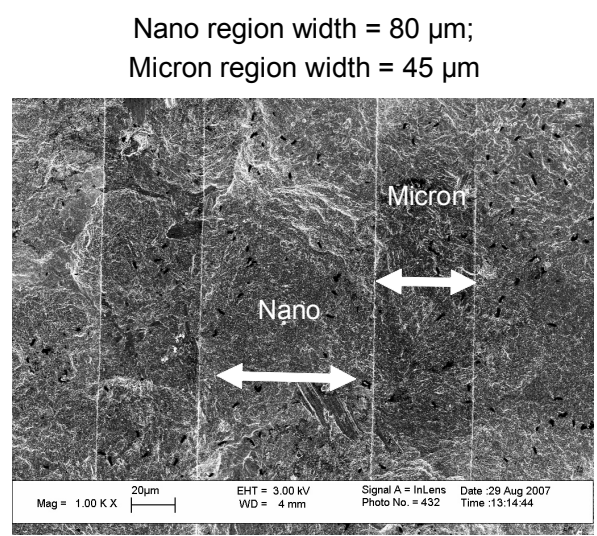

(a) Patterned Ti substrates $($ scale bar $=20 \mu \mathrm{m})$

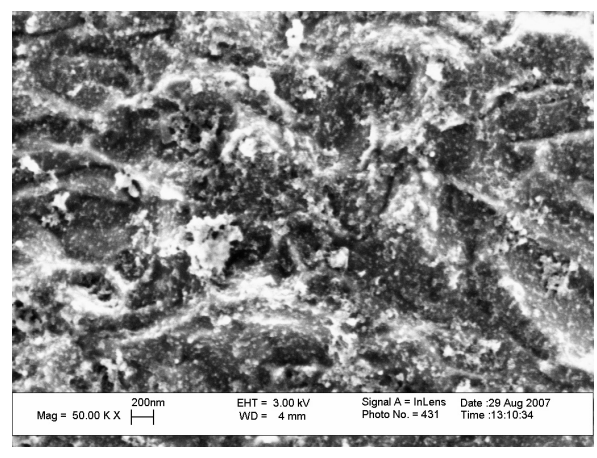

(b) Micron region on patterned Ti substrate (scale bar $=200 \mathrm{~nm}$ )

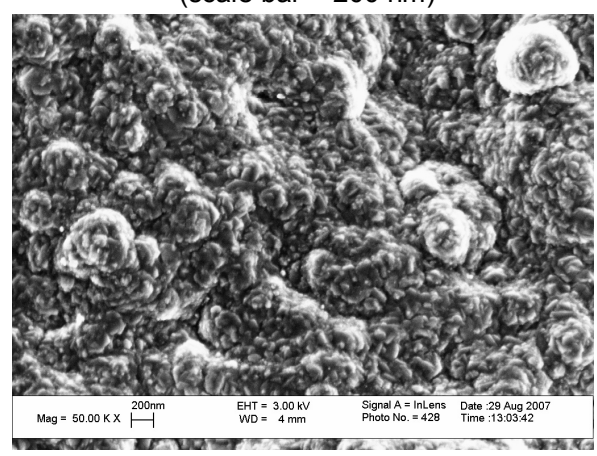

(c) Nano region on patterned Ti substrate $($ scale bar $=200 \mathrm{~nm})$

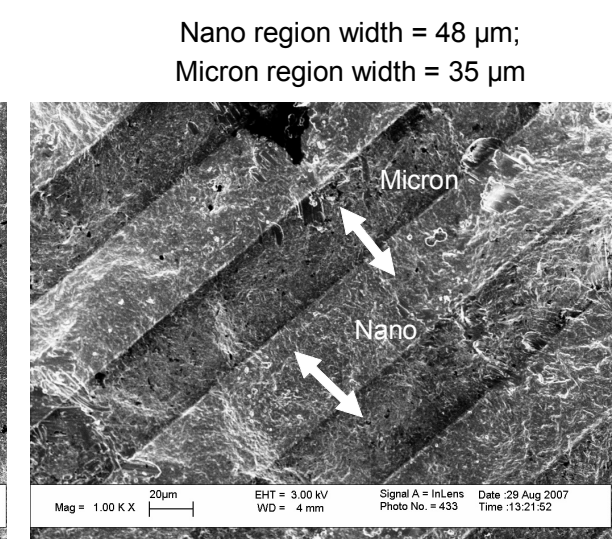

(d) Patterned Ti substrates (scale bar $=20 \mu \mathrm{m}$ )

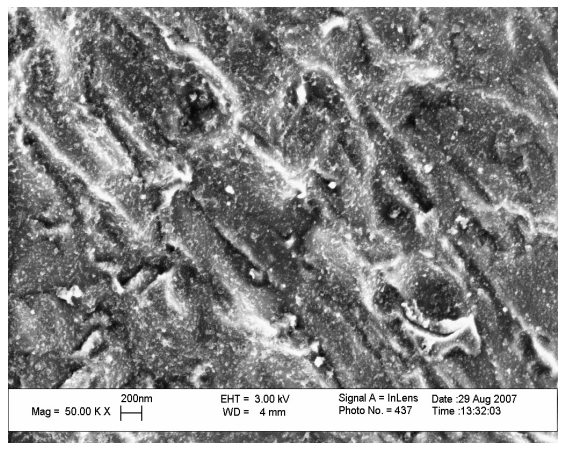

(e) Micron region on patterned Ti substrate $($ scale bar $=200 \mathrm{~nm})$

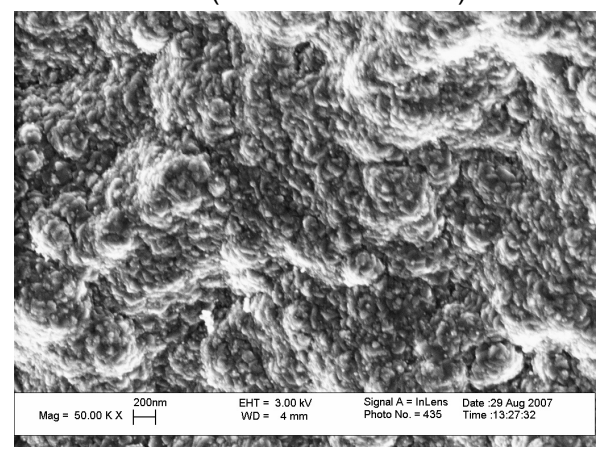

(f) Nano region on patterned Ti substrate $($ scale bar $=200 \mathrm{~nm})$ 


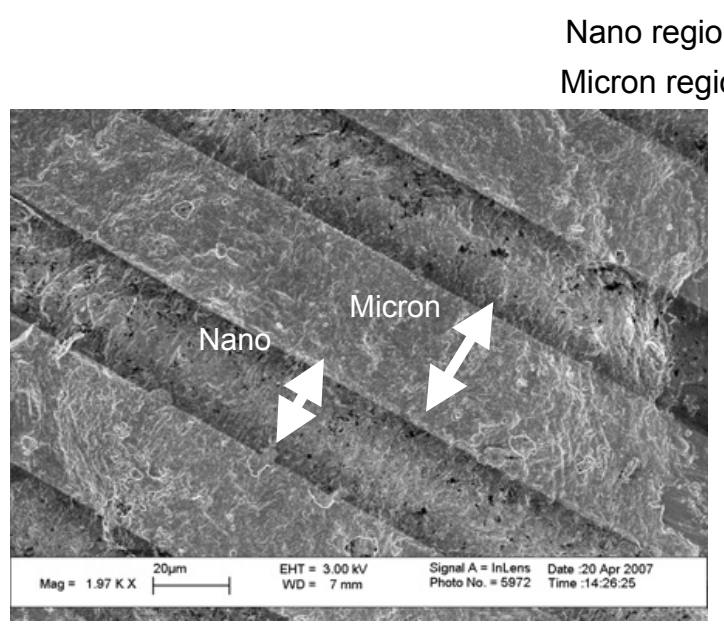

(g) Patterned Ti substrates

(scale bar $=20 \mu \mathrm{m})$

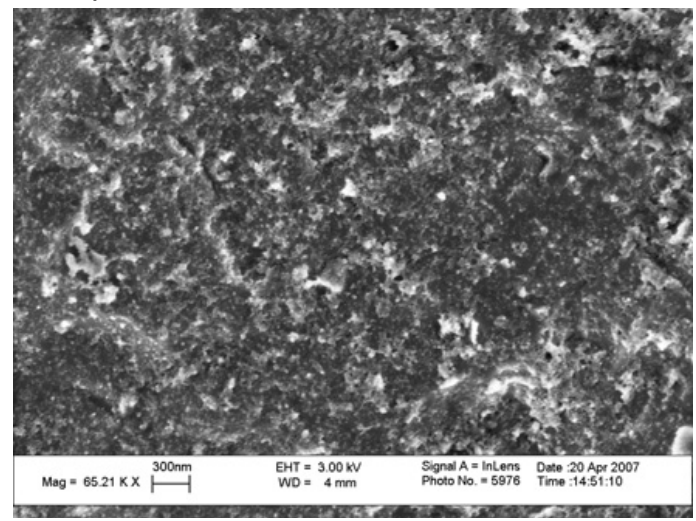

(h) Micron region on patterned Ti substrate $($ scale bar $=300 \mathrm{~nm})$

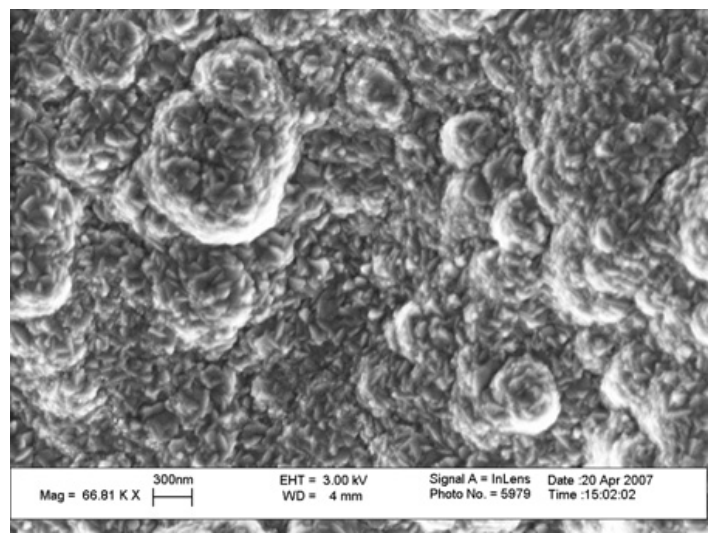

(i) Nano region on patterned Ti substrate

$($ scale bar $=300 \mathrm{~nm})$

Figure 2 Scanning electron microscopy images of the $(\mathbf{a}, \mathbf{d}, \mathbf{g})$ patterned Ti substrates, $(\mathbf{b}, \mathbf{e}, \mathbf{h})$ micron region of the patterned Ti substrate, and (c, $\mathbf{f}, \mathbf{i})$ nano region of the patterned Ti substrate. Nano regions on patterned substrates were created using electron beam deposition and possessed an increased nanometer surface roughness compared with the micron region of the patterned Ti substrates. Bars $=(\mathbf{a}, \mathbf{d}, \mathbf{g}) 20 \mu \mathrm{m}$; (h, i) $300 \mathrm{~nm}$; (b, c, e, f) $200 \mathrm{~nm}$.

compared to the entire unmodified micron rough substrates and the entire modified nano rough substrates (Figure 8). Although the osteoblasts elongated both parallel and perpendicular relative to the patterns, they were confined within the individual regions suggesting the possibility of aligned proliferation (and possibly mineral deposition) during long term experiments. There was no alignment or specific orientation of osteoblasts identified on the substrates containing no patterns indicating that the presence of linear features can control the direction and behavior in which osteoblasts adhere. Furthermore, differences in osteoblast morphology were also observed on each of the varying patterned Ti substrates examined. More specifically, decreasing the width of the nano rough modified region resulted in decreased osteoblast spreading (Figure 8). In other words, osteoblast morphology on the Ti patterned substrates containing the smallest nano rough region were more round and had less diffuse f-actin filaments. This may explain in part why there was a decrease in osteoblast adhesion on the nano rough regions that had smaller nano region widths as indicated in Figure 7.

Alignment and morphology of osteoblasts on patterned Ti substrates after 4 hours was further verified using SEM (Figure 9). Whether cells adhered on the micron or nano rough Ti region of the patterned substrates, osteoblast elongation and alignment were observed. In fact, images clearly revealed that the filopodia extensions were extending from the cell and remained within the region from which they were located. Such results indicated that osteoblasts were recognizing (possibly through altered protein interactions) the different surface roughness on these patterned Ti substrates. 


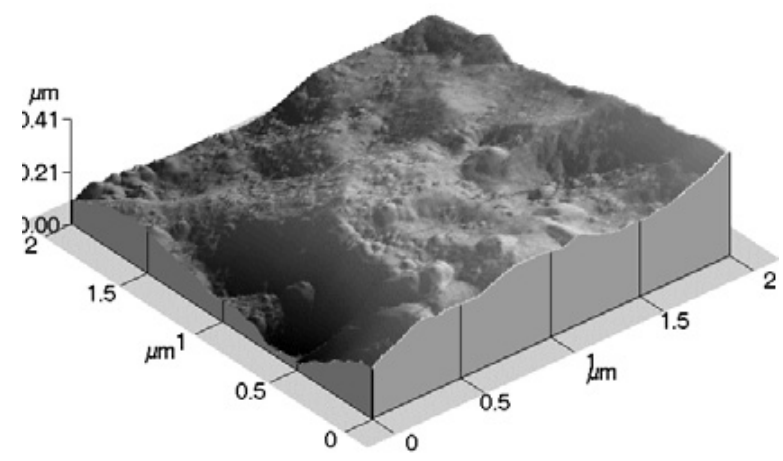

(a) Micron rough region

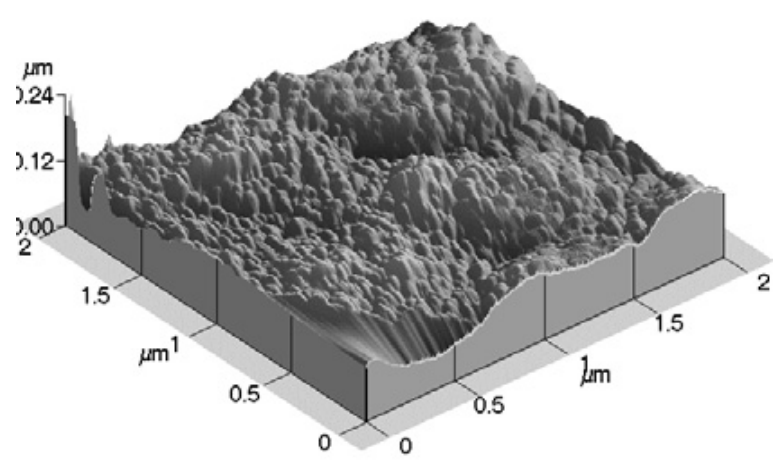

(b) Nano rough region

Figure 3 Atomic force microscopy images demonstrating the topographical differences in the (a) unmodified, micron rough region and the (b) modified, nano rough region on the patterned Ti substrates.

\section{Discussion}

In order to be classified as a nanomaterial, a material must have at least one dimension (whether it is the basic structural unit, grain size, particle size, diameter, or surface-coating thickness) in the range of $100 \mathrm{~nm}$ or less.
Current orthopedic devices consist of conventional metal and metal alloys that have grain sizes greater than $100 \mathrm{~nm}$ (Kaplan et al 1994; Webster and Ahn 2006). Although the surfaces of these conventional implants are not equivalent to the surface roughness of natural bone, these devices are

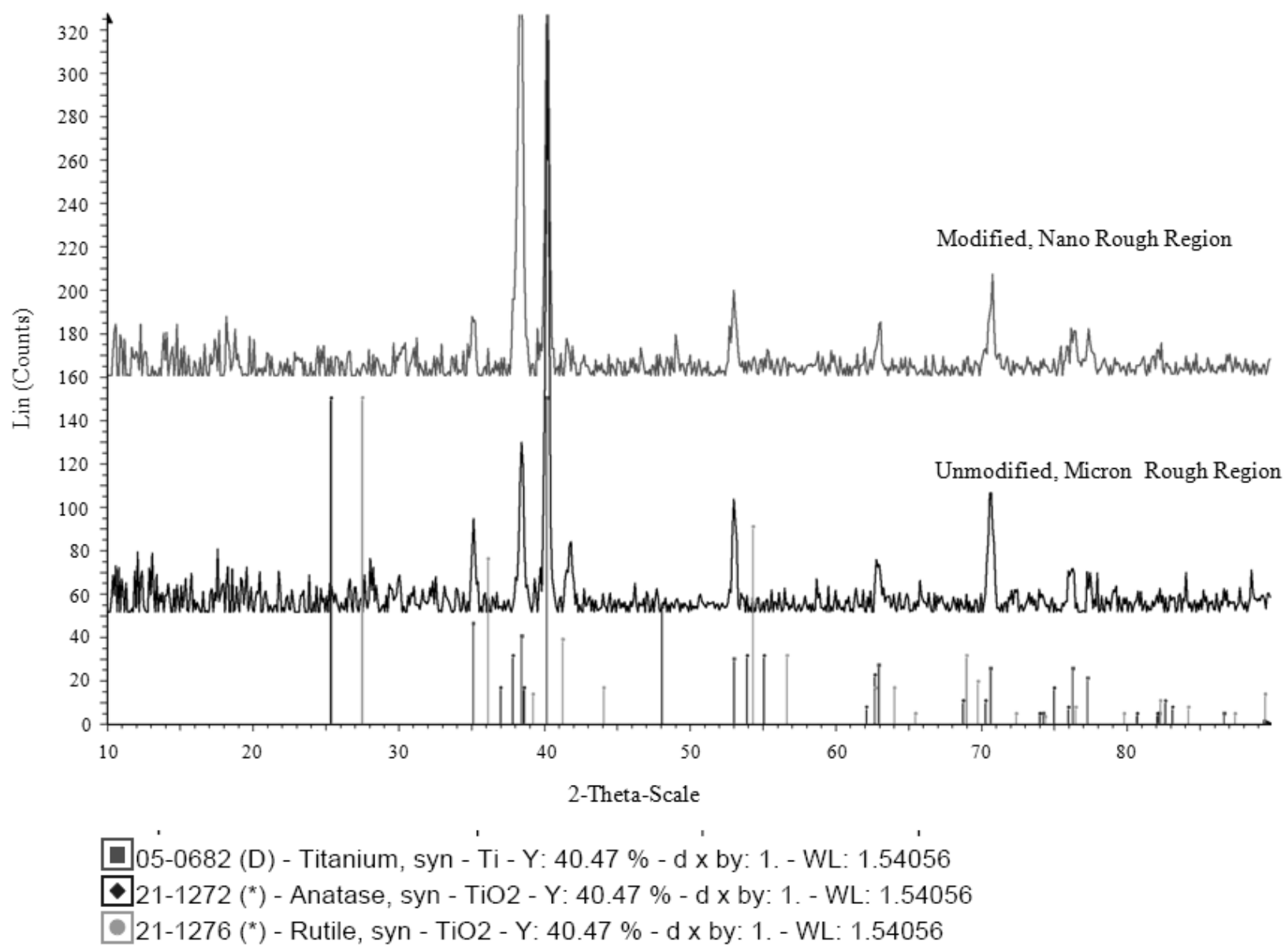

Figure $4 \mathrm{X}$-ray diffraction images of the two regions on the patterned substrates. The graph on the top represents the modified, nano region while the graph on the bottom represents the original, unmodified $\mathrm{Ti}$ surface. The graph indicates the presence of rutile $\mathrm{TiO}_{2}$ on the conventional region while the nano rough region contains anatase $\mathrm{TiO}_{2}$. 


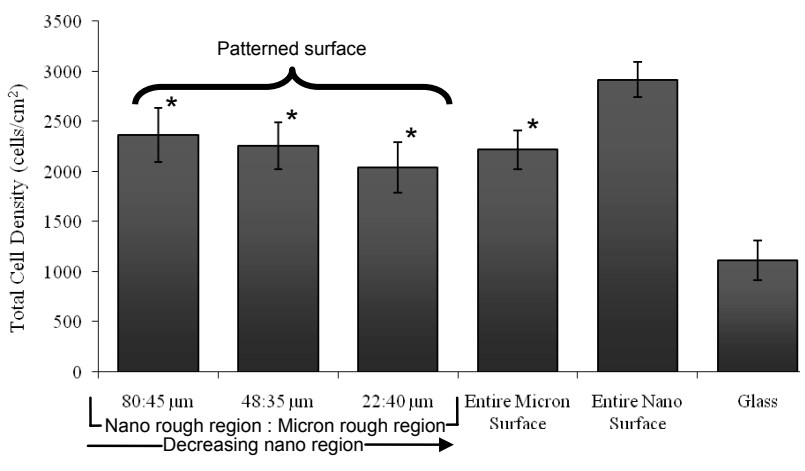

Figure 5 Increased total osteoblast adhesion on the entire nano rough Ti surface after four hours. Notes: Data are mean \pm SEM; $n=3$; ${ }^{*} p<0.0$ I compared to the entire nano Ti surface. Indicated on this graph is the total number of cells on each substrate irrespective of location of attachment.

valued for their biocompatibility (Long and Rack 1998) and ability to improve the quality of life for patients. However, these orthopedic prostheses are only a temporary solution in part because of insufficient bonding to juxtaposed bone (Bloebaum et al 1993; Toni et al 1994; Ichikawa et al 1996; Anselm et al 1999). As a result, loosening of the implant occurs and leads to early failure of these orthopedic implant devices. Surface properties of the material are one factor that may explain why there is a lack of osseointegration upon implantation. The current micron surfaces are not providing optimal conditions for initial cellular interactions. Consequently, creating surfaces that induce a more comparable and compatible environment for osteoblasts, and thereby facilitate new bone ingrowth, is ideal.

Research is currently aimed at using nanotechnology as a way of specifically mimicking the size of the grain boundaries seen on natural bone. Bone is a naturally nano rough surface that consists of nanosize collagen and hydroxyapatite molecules. As a result, producing nano rough surfaces on these implants is more favorable for osteoblast growth than the current conventional implant surfaces since osteoblasts are familiar with this type of surface structure. In fact, research groups have already shown that there is an increase in osteoblast adhesion and function on nanophase materials compared to conventional materials. Such nanophase materials include metals (de Oliveira and Nanci 2004; Webster and Ejiofor 2004; Yao et al 2005; Ward and Webster 2007), ceramics (Webster et al 1999a, 1999b, 2000a; Karlsson et al 2003; Price et al 2003a; Gutwein and Webster 2004), polymers (Kay et al 2002; Price et al 2003b; Washburn et al 2004; Palin et al 2005), and composites (Kay et al 2002; Price et al 2003b; Webster and Smith 2005). However, one dimension in research that has yet to be examined is studying how collagen and

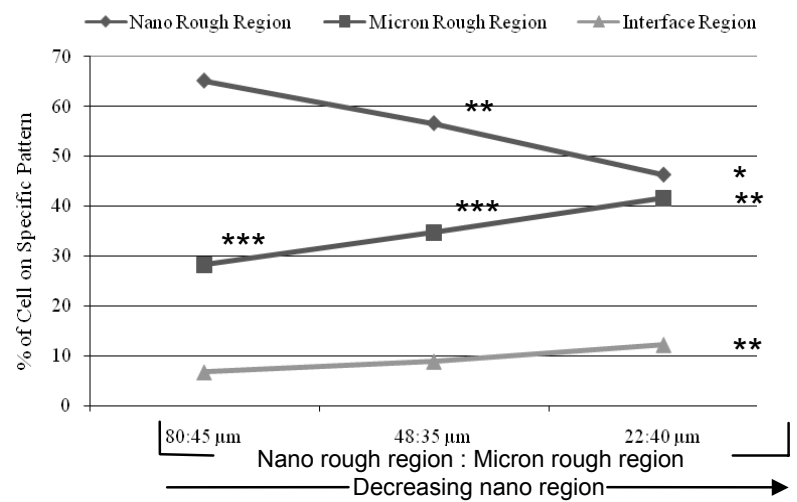

Figure 6 Greater osteoblast adhesion on the Ti nano rough region regardless of pattern width after four hours.

Notes: Data are mean \pm SEM; $\mathrm{n}=3 ;{ }^{*} \mathrm{p}<0.01$ and ${ }^{* *} \mathrm{p}<0.05$ compared with the $80: 45 \mu \mathrm{m}$ grid pattern on the respective regions; $* * * \mathrm{p}<0.01$ compared with the nano region on the respective samples. Indicated on this graph are the percent of cells adhering to the specific pattern of each substrate. This data was collected from the same experiments as those completed in Figure 5.

hydroxyapatite orientation in long bones as mimicked on Ti affects osteoblast performance. It has been shown that collagen spontaneously forms fibrils of aligned nanoscale protein helices (Holmgren et al 1998; Lodish et al 2000; Taton 2001). Since research is providing evidence that surfaces which replicate natural bone structures produce an environment more conducive for osteoblast function and, thus, bone ingrowth, the impact aligned nano features can have on osteoblasts is of particular interest.

This study provided evidence that creating surfaces with linearly aligned patterns has an effect on osteoblast response. First, there was an increase in total osteoblast adhesion on the entire nano rough Ti surfaces. Although this data confirmed that reported in other studies as mentioned above, the technique used to create the nano rough

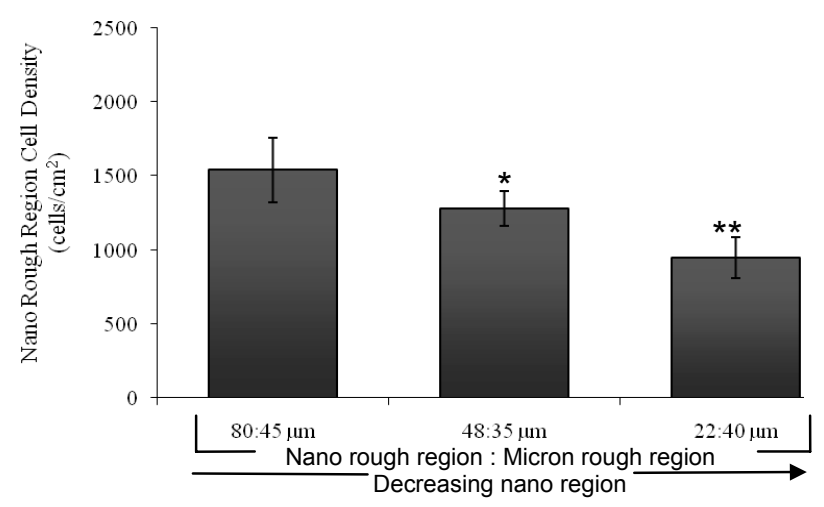

Figure 7 Decreasing the width of the nano rough region on patterned Ti substrates decreased osteoblast adhesion after four hours.

Notes: Data are mean \pm SEM; $n=3 ;{ }^{*} p<0.05$ and **p $<0.01$ compared with the $80: 45 \mu \mathrm{m}$ grid pattern. Indicated on this graph are the percent of cells adhering specifically to the nano rough region of each substrate. This data was collected from the same experiments as those completed in Figures 5 and 6. 


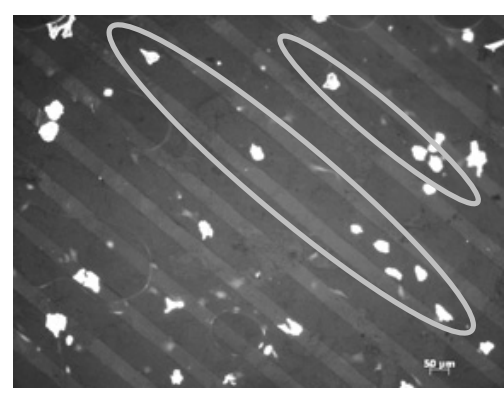

(a) 80 (nano):45 (micron) $\mu \mathrm{m}$

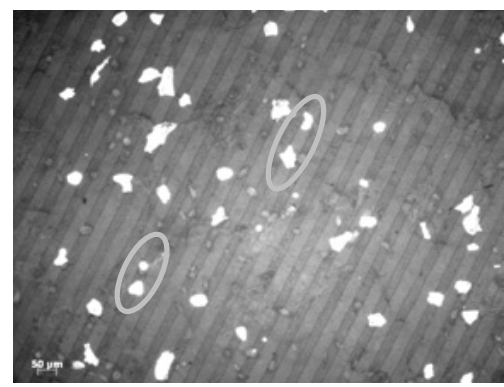

(c) 22 (nano):40 (micron) $\mu \mathrm{m}$

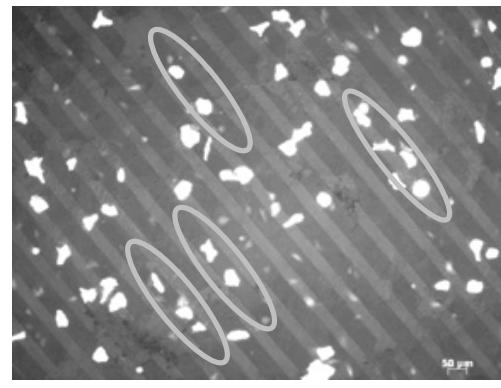

(b) 48 (nano):35 (micron) $\mu \mathrm{m}$

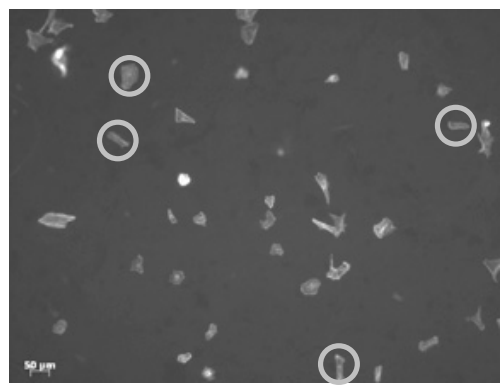

(d) complete micron rough

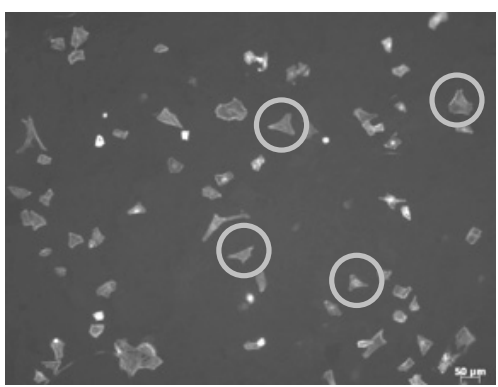

(e) complete nano rough

Figure 8 Fluorescent microscopy images of osteoblasts on (a-c) patterned Ti substrates, (d) complete micron rough Ti substrates, and (e) complete nano rough Ti substrates after four hours. Osteoblast alignment was detected on the patterned substrates when compared with the entire micron and nano rough Ti substrates. Among the patterned substrates, osteoblast morphology varied. As the nano region width decreased, cells became more round and less diffuse. Magnification $=10 \times$. Nano patterned regions appear darker.

features on Ti differed. More specifically, electron beam evaporation is a more versatile, efficient, and simple technique compared with other methods, such as anodization, particle compaction, and chemical etching. Electron beam evaporation allows for the easy control of the deposited thickness and, thus, grain size which in turn allows for better control of the nano rough characteristics. Electron beam evaporation can also coat unique implant geometries where other techniques fail. In this study, electron beam evaporation was used to create linear patterns of alternating micron and nano rough regions. These topographical differences were demonstrated by SEM and AFM, in which the modified regions clearly displayed nanoscale features. Typically, nano rough surfaces have been shown to have higher rms values than micron rough surfaces. However, on these patterned substrates, AFM revealed the opposite, a higher rms value for the micron rough regions. Looking more closely at the original Ti surface, large dips and ridges are present resulting in large height measurements compared to the modified regions. This underlying roughness is creating higher rms values for the micron rough regions. On the other hand, the modified, coated regions are masking and smoothing out the roughness of the original surface with nano bumps creating smaller rms values. Clearly, through electron beam deposition, surfaces were created with two distinct topographies, one representing micron rough features and the other representing nano rough features.

Second, this study further provided evidence that creating aligned patterns of alternating nano rough and micron rough regions resulted in the initial alignment of osteoblasts on these surfaces. This led to early indications that the 

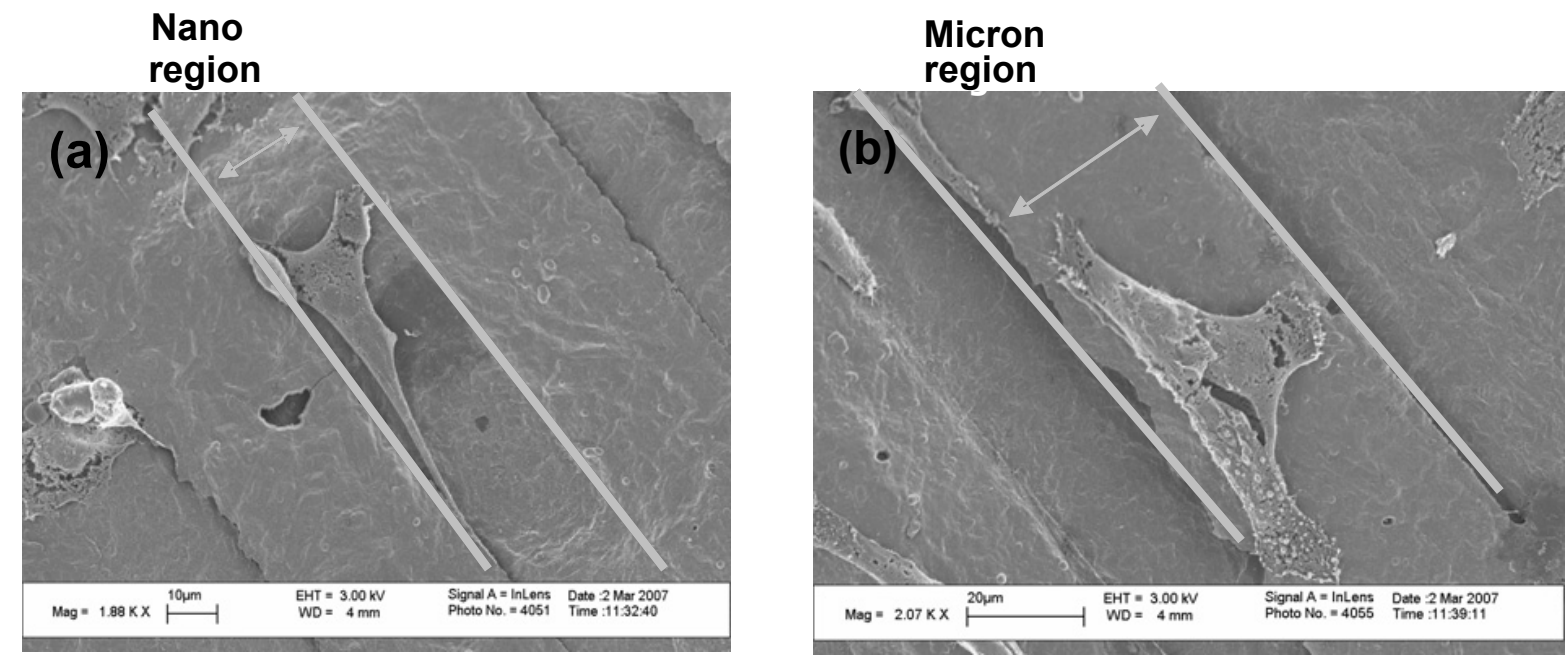

\section{Nano region}

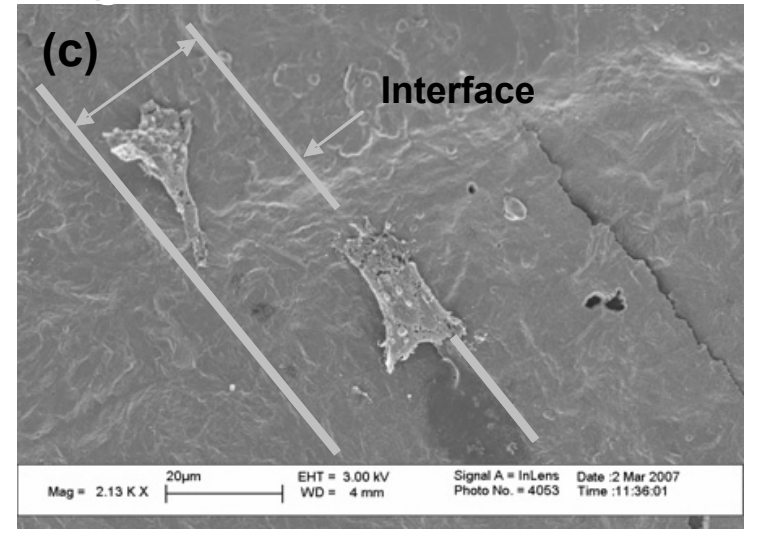

Figure 9 Scanning electron microscopy of osteoblasts adhering on patternedTi substrates ( $22 \mu \mathrm{m}$ nano rough region: $40 \mu \mathrm{m}$ micron rough region) after four hours. Osteoblast alignment and elongation was observed despite the region of attachment. Filopodia extensions were maintained within the region where the osteoblast was located. Notes: Bars $=(\mathbf{a}) 10 \mu \mathrm{m}$; $(\mathbf{b}$ and c) $20 \mu \mathrm{m}$.

orientation of collagen and hydroxyapatite in long bones may play an important role for osteoblast adhesion, function, and growth. Third, this study provided evidence of greater osteoblast adhesion on the modified nano rough region of these patterned Ti substrates. Directed osteoblast adhesion on the nano rough Ti regions of the patterned substrates may be explained by the fact that they contained greater particle boundaries at the surface due to smaller particle size. Such data indicates that osteoblasts are not only aligning to the linear patterns but they are also reacting and responding to the varying surface features created through the use of electron beam techniques. These surfaces created by this deposition process possess not only a different topography but also a different crystallinity when compared with the conventional surfaces that have not been altered by deposition. More specifically, the nano rough regions of the patterned substrates contained anatase $\mathrm{TiO}_{2}$ whereas the micron rough regions lacked the presence of anatase $\mathrm{TiO}_{2}$. It has been shown through previous research that the properties of anatase $\mathrm{TiO}_{2}$ enhance the ability to induce bone-like hydroxyapatite formation when compared with rutile $\mathrm{TiO}_{2}$ (Tang et al 2004). This, therefore, explains in part why osteoblasts were adhering more to the nano rough regions on these patterned substrates. As a result, it can be concluded that the difference in osteoblast adhesion on the varying regions may be due to both a crystalline and topographical effect. The fact that osteoblasts were adhering more to the modified, nano rough regions indicates that they favor surfaces that are more like that of natural bone (nano rough), and as a result, better conditions are being derived to create optimal interactions between the device and its surroundings. Although clearly much more testing is needed, this may lead to more sufficient bonding to juxtaposed bone and therefore minimize the early failure of orthopedic implants. Another possible explanation 
for better osteoblast adhesion on the nano rough regions of the patterned $\mathrm{Ti}$ substrates is their optimal interactions with proteins. Research groups have already indicated the importance of proteins in mediating osteoblast adhesion (such as fibronectin and vitronectin) on nanophase materials (Webster et al 2000b, 2001; Yang et al 2003). Therefore, protein responses to the patterned $\mathrm{Ti}$ substrates may be responsible for the optimal osteoblast behavior observed on these materials.

Lastly, this study was interested in determining if early functions of osteoblasts were affected by the size of the aligned patterns. Results suggested that there is some optimal pattern dimension of nano features to which osteoblasts prefer to function. In other words, a threshold exists in which osteoblasts do not attach and spread properly. More specifically, data from this study revealed that decreasing the width of the nano rough region on these patterned substrates results in decreased osteoblast adhesion on nano rough regions. This could be in part due to the size of osteoblasts (20-30 $\mu \mathrm{m}$ in diameter). When nano rough regions become too small for osteoblasts to comfortably attach to and extend, they acquire a round morphology and their f-actin filaments are less diffuse. Therefore, aligned nano features are a promising new technique for enhancing osteoblast adhesion and function, under which there are optimal conditions at which this can be achieved.

\section{Conclusions}

The present study provided the first evidence of improved osteoblast adhesion and morphology on Ti substrates with linearly aligned nano features similar to the size and orientation of collagen and hydroxyapatite in long bones. In creating these patterns, this study also indicated that the dimensions of these patterns can effect the alignment and adhesion of osteoblasts on these surfaces. Thus, this study demonstrates that the formation of micron patterns of nano features as prepared by e-beam evaporation on titanium should be further studied to increase the longevity of orthopedic implants.

\section{Acknowledgments}

The authors wish to acknowledge the Coulter Foundation and Department of Veterans Affair Pre-Doctoral Associated Health Rehabilitation Research Fellowship for funding. The authors also wish to acknowledge the Microelectronics Facility, the Leduc Bioimaging Facility at Brown University, and Mr. Anthony McCormick for his help with SEM and AFM.

\section{References}

[AAOS] American Academy of Orthopedic Surgeons. 2006a. Number of total hip replacement procedures [online]. Accessed August 31, 2006. URL: http://www.aaos.org/Research/stats/Total\%20Hip\%20Replace ment $\% 20$ Chart.pdf.

[AAOS] American Academy of Orthopedic Surgeons. 2006b. Number of total knee replacement procedures. Accessed August 31, 2006. URL: http://www.aaos.org/Research/stats/Total\%20Knee\%20Replacemen t\%20Chart.pdf.

[AAOS] American Academy of Orthopedic Surgeons. 2006c. Total knee and hip replacement surgery projections show meteoric rise by 2030 . Accessed August 31, 2006. URL: http://www6.aaos.org/news/Pemr/ press_release.cfm? prnumber $=442$.

Anselm Wiskott HW, Belser UC. 1999. Lack of integration of smooth titanium surfaces: a working hypothesis based on strains generated in the surrounding bone. Clin Oral Impl Res, 10:429-44.

Anselme K. 2000. Osteoblast adhesion on biomaterials. Biomaterials, 21:667-81.

Bloebaum RD, Dupont JA. 1993. Osteolysis from a press-fit hydroxyapatitecoated implant. J Arthroplasty, 8:195-202.

Brunski JB. 1991. Influence of biomechanical factors at the bone-biomaterials interface. In: Davies JE (ed). The bone-biology materials interface. Toronto: Univ of Toronto Pr, pp. 391-404.

de Oliveira PT, Nanci A. 2004. Nanotexturing of titanium-based surfaces upregulates expression of bone sialoprotein and osteopontin by cultured osteogenic cells. Biomaterials, 25:403-13.

Gutwein LG, Webster TJ. 2004. Increased viable osteoblast density in the presence of nanophase compared to conventional alumina and titania particles. Biomaterials, 25:4175-83.

Holmgren SK, Taylor KM, Bretscher LE, et al. 1998. Code for collagen's stability deciphered. Nature, 392:666-67.

Ichikawa T, Hirota K, Kanitani H, et al. 1996. Rapid bone resorption adjacent to hydroxyapatite-coated implants. J Oral Implantol, 22:232-35.

Jayaraman M, Meyer U, Buhner M, et al. 2004. Influence of titanium surfaces on attachment of osteoblast-like cells in vitro. Biomaterials, 25:625-31.

Kaplan FS, Hayes WC, Keaveny TM, et al. 1994. Form and function of bone. In: Simon SP (ed). Orthopedic basic science. Ohio: American Academy of Orthopedic Surgeons, pp. 127-85.

Karlsson M, Palsgard E, Wilshaw PR, et al. 2003. Initial in vitro interaction of osteoblasts with nano-porous alumina. Biomaterials, 24:3039-46.

Kay S, Thapa A, Haberstroh KM, et al. 2002. Nanostructured polymer/ nanophase ceramic composites enhance osteoblast and chondrocyte adhesion. Tissue Eng, 8:753-61.

Liu H, Webster TJ. 2007. Nanomedicine for implants: A review of studies and necessary experimental tools. Biomaterials, 28:354-69.

Lodish H, Berk A, Zipursky, et al. 2000. Integrating cells into tissues. In: Matsodavia P (ed). Molecular cell biology. $4^{\text {th }}$ ed. New York: W. H. Freeman and Company.

Long M, Rack HJ. 1998. Titanium alloys in total joint replacement - a materials science perspective. Biomaterials, 19:1621-39.

Palin E, Liu H, Webster TJ. 2005. Mimicking the nanofeatures of bone increases bone-forming cell adhesion and proliferation. Nanotech, 16:1828-35.

Price RL, Gutwein LG, Kaledin L, et al. 2003a. Osteoblast function on nanophase alumina materials: influence of chemistry, phase, and topography. J Biomed Mater Res A, 67:1284-93.

Price RL, Waid MC, Haberstroh KM, et al. 2003b. Selective bone cell adhesion on formulations containing carbon nanofibers. Biomaterials, 24:1877-87.

Rho JY, Kuhn-Spearing L, Zioupos P. 1998. Mechanical properties and the hierarchal structure of bone. Med Eng Phys, 20:92-102.

Tang G, Zhang R, Yan Y, et al. 2004. Preparation of porous anatase titania film. Mater Letter, 58:1857-60.

Taton TA. 2001. Boning up on biology. Nature, 412:491-2. 
Toni A, Lewis CG, Sudanese A, et al. 1994. Bone demineralization induced by cementless alumina-coated femoral stems. J Arthroplasty, 9:435-44.

Ward BC, Webster TJ. 2007. Increased functions of osteoblasts on nanophase metals. Biomaterials, 27:574-78.

Washburn NR, Yamada KM, Simon CG, et al. 2004. High-throughput investigation of osteoblast response to polymer crystallinity: influence of nanometer-scale roughness on proliferation. Biomaterials, 25:1215-24.

Webster TJ, Siegel RW, Bizios R. 1999a. Osteoblast adhesion on nanophase ceramics. Biomaterials, 20:1221-27.

Webster TJ, Siegel RW, Bizios R. 1999b. Design and evaluation of nanophase alumina for orthopedic/dental applications. Nano Struct Mater, 12:983-86.

Webster TJ, Ergun C, Doremus RH, et al. 2000a. Enhanced functions of osteoblasts on nanophase ceramics. Biomaterials, 21:1803-10.

Webster TJ, Ergun C, Doremus RH, et al. 2000b. Specific proteins mediate enhanced osteoblast adhesion on nanophase ceramics. J Biomed Mater Res, 51:475-83.

Webster TJ. 2001. Nanophase ceramics: the future orthopedic and dental implant material. Adv Chem Eng, 27:125-66.
Webster TJ, Schadler LS, Siegel RW, et al. 2001. Mechanisms of enhanced osteoblast adhesion on nanophase alumina involve vitronectin. Tissue Eng, 7:291-301.

Webster TJ, Ejiofor JU. 2004. Increased osteoblast adhesion on nanophase metals: Ti, Ti6Al4V, and CoCrMo. Biomaterials, 25:4731-39.

Webster TJ, Smith TA. 2005. Increased osteoblast function on PLGA composites containing nanophase titania. J Biomed Mater Res A, 74:677-86.

Webster TJ, Ahn ES. 2006. Nanostructured biomaterials for tissue engineering bone. Adv Biochem Engin/Biotechnol, 103:275-308.

Yang Y, Glover R, Ong JL. 2003. Fibronectin adsorption on titanium surfaces and its effect on osteoblast precursor cell attachment. Col Surf B: Biointerfs, 30:291-97.

Yao C, Slamovich EB, Qazi JI, et al. 2005. Improved bone cell adhesion on ultrafine grained titanium and Ti-6Al-4V. Ceramic Transactions, 159:239-46. 
